\title{
Trends in Managed Care Pharmacy: Preparing for the Future
}

$\mathrm{T}$

he mandate of managed care organizations (MCOs) is to provide quality health care while responsibly managing health care costs. Managed care has brought significant change to the way health care services are provided, most notably by integrating many care components and linking them financially and clinically. It has also produced changes in how health care providers and patients participate in the system and relate to each other.

As managed care has grown in the past decade, employers, health care providers, and patients have demanded more autonomy, choice, and flexibility. Along with choice, however, comes the potential for increasing costs and a need to determine the bearer of those costs. External factors such as new technology, procedures, and drugs, combined with changing member demographics and increasing numbers of Medicare and Medicaid patients, have also had an impact on costs. These trends suggest the need for a reassessment of the direction managed care organizations must take to both succeed in business and focus on patient needs.

One method for improving both patient care and the financial outlook of MCOs involves making better use of the vast amounts of clinical and financial data available within managed care systems, especially in the pharmacy area.
Addressing the demands of consumers, physicians, and employers requires an accurate assessment of costs and clinical outcomes. Many organizations are not yet using an integrated approach that includes several, if not most, components of care. Pharmacy departments are in a unique position to view cost and clinical outcomes across the continuum of care, from primary care visits to hospital inpatient care and post-hospitalization, because they touch the patient at each stage of care. Pharmacy departments can take the initiative in developing models for improved patient and financial outcomes by using the information available for the benefit of the entire organization. This may mean going directly to the information-systems department, identifying subsets of patients who use significant resources, and examining ways to improve resource use to optimize outcomes. It may mean conducting studies of particular patient populations. Regardless of the tactics used, pharmacy can often be the focal point to highlight important clinical and economic issues and the results of interventions.

This article reflects on several key managed care evolutionary trends and some challenges for the future. Managed care data cited were supplied by SMG Marketing Group, Inc., and published in the Aventis Managed Care Digest Series, a central source of information about all aspects of managed care. ${ }^{1}$ In addition, this paper includes examples demon- strating how pharmacists can use information available to them within their own organizations to highlight areas for improvement in patient intervention while also decreasing costs for the entire health plan.

\section{Growth of Managed Care Organizations}

There has been an overall increase in managed care penetration in recent years, with 104.6 million persons, $37.9 \%$ of the U.S. population, in health maintenance organizations (HMOs) in 1999 (see Figure 1, page 106). Of that total, 18.5 million persons were enrolled in pointof-service (POS) plans, which provide more flexibility in choice of providers. As a result of consumer demand for choice, interest in preferred provider organizations (PPOS) and in POS plans has remained strong. Employers also appreciate that in these programs consumers themselves bear at least some of the economic costs of the choices they make.

Membership demographics in managed care organizations have also been affected by a significant increase in Medicare and Medicaid patients (see Table 1, page 106). In 1998 HMO enrollees included 6.5 million Medicare beneficiaries and 9.8 million Medicaid beneficiaries. Those numbers rose to 6.7 million for Medicare and 11.3 million for Medicaid in 1999, even though some Medicare risk plans withdrew from the

\section{Author}

JOSEPH EICHENHOLZ, M.A., is Senior Vice President, Quadrant HealthCom, Inc., Chatham, NJ.

AUTHOR CORRESPON DENCE: Joseph Eichenholz, M.A., Senior Vice President, Quadrant HealthCom, Inc., 26 Main Street, Chatham, NJ 07927; Tel: 973-701-2782;

Fax: 973-701-8894; E-mail: joe.eichenholz@qhc.com.

Copyright@ 2001 Academy of Managed Care Pharmacy, Inc. All rights reserved. 


\section{National HMO Penetration}

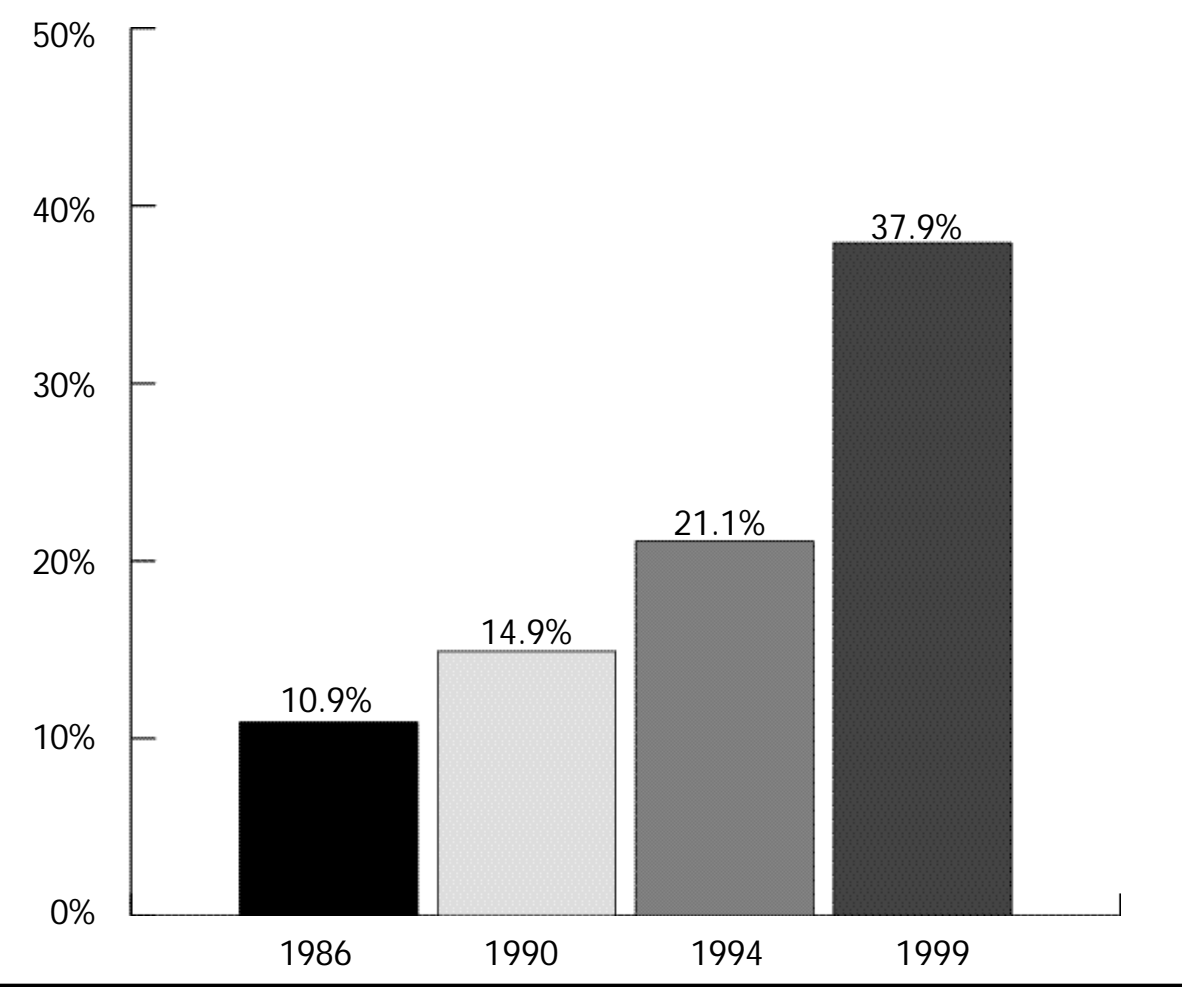

Source: Data from SM G M arketing Group, Inc., Chicago, IL, as published in Managed Care Digest Series 2000. Parsippany, NJ: Aventis Pharmaceuticals, 2000.

\begin{tabular}{c|c|c|c|c}
\hline & \multicolumn{2}{c}{ Medicare and Medicaid HMO Enrollment, 1986-1999 } \\
\hline & \multicolumn{2}{c}{$\begin{array}{c}\text { Number of } \\
\text { Enrollees (Thousands) }\end{array}$} & \multicolumn{2}{c}{$\begin{array}{c}\text { Number of HMOs Accepting } \\
\text { Government Beneficiaries }\end{array}$} \\
\hline 1986 & Medicare & Medicaid & Medicare & Medicaid \\
\hline 1990 & 1,421 & 602 & 332 & 159 \\
\hline 1994 & 3,896 & 842 & 211 & 88 \\
\hline 1998 & 6,080 & 2,665 & 223 & 146 \\
\hline 1999 & 6,698 & 9,795 & 335 & 267 \\
\hline
\end{tabular}

Source: Data from SM G Marketing Group, Inc., Chicago, IL, as published in M anaged Care Digest Series 2000. Parsippany, NJ: Aventis Pharmaceuticals, 2000.

program. The growth in these population segments within HMOs has important implications. It reflects continuing interest by federal and state governments to cap their own financial risk while assuring availability of care. For health plans, the Medicare and Medicaid populations represent a potential source of new members that does not require attracting members away from other health plans. Yet the difficulties in providing care for these members, assuming adequate communication, and managing financial risk, are becoming more apparent each year.
The increase in the number of older Americans has also changed HMO membership demographics, affecting service and resource use. As the general population ages, there is an increasing need for treatment of chronic age-related diseases, such as cardiovascular disease, type-2 diabetes, arthritis, and osteoporosis. The addition to HMOs of members who are older and sicker challenges both the clinical protocols and the fiscal viability of today's MCOs.

\section{HMO Trends}

\section{HMO Pharmacy and Utilization}

Increased HMO growth and enrollment has also brought increased utilization. Over the past five years, ambulatory visits and physician encounters rose for all enrollees (non-Medicare, Medicare, and Medicaid). Physician encounters for Medicare HMO members almost doubled during the 1990s. Prescription use also increased significantly between 1988 and 1999 . The average number of prescriptions for non-M edicare members per year increased from 4.9 in 1988 to 7.1 in 1999. Pharmacy expenditures increased as well. Average ingredient costs rose from $\$ 11.50$ in 1988 to $\$ 28.35$ in 1999. As a result, pharmacy premiums for both individuals and families grew at an average annual compounded rate of $2.36 \%$ between 1988 and 1999. Pharmacy expenses as a percentage of total operating expenses grew from $10 \%$ to $13.4 \%$ during the same period (see Table 2 and Figure 2, both on page 107).

Coinciding with increases in pharmacy costs, however, are significant decreases in the use of hospital care. Hospital admissions per 1,000 non-M edicare patients decreased $21.7 \%$ between 1990 and 1998; length-of-stay decreased $26 \%$. For Medicare patients, however, hospital admissions increased $10.5 \%$ between 1994 and 1998. Table 3, page 108, illustrates utilization rates by payor type for 1998 and 1999. Interestingly, increased pharmacy costs correlate with decreased hospitalization. Specifically, the significant increase in prescriptions for non- 


\section{Drug Costs as a Percentage of HMO Operating Expenses}

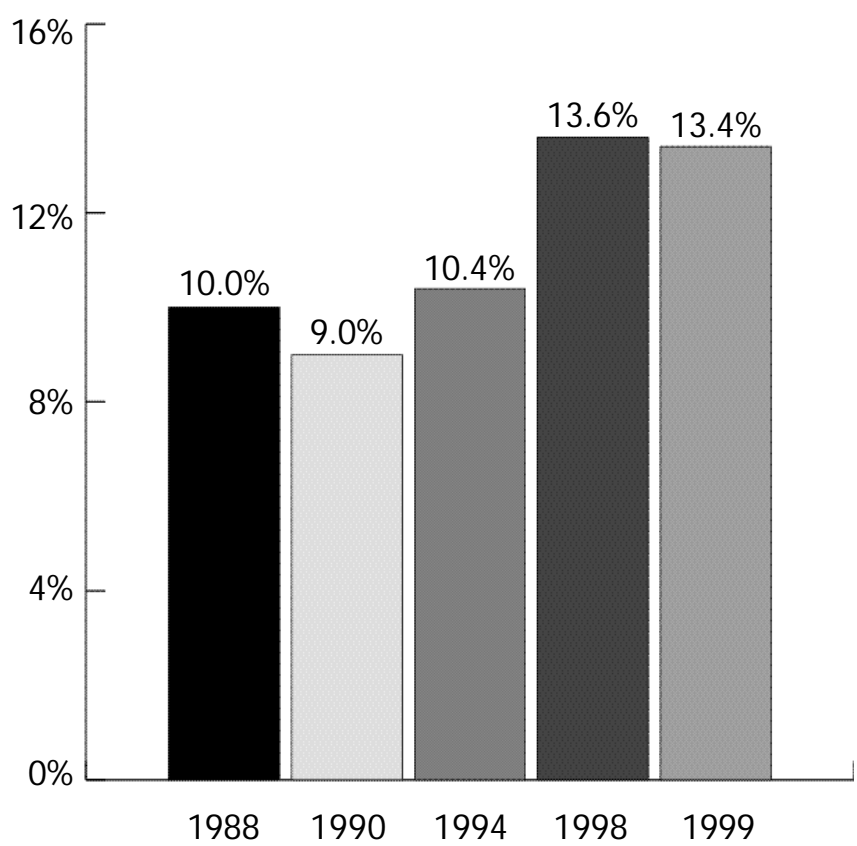

\section{Percentage of HMOs using Closed Formularies}

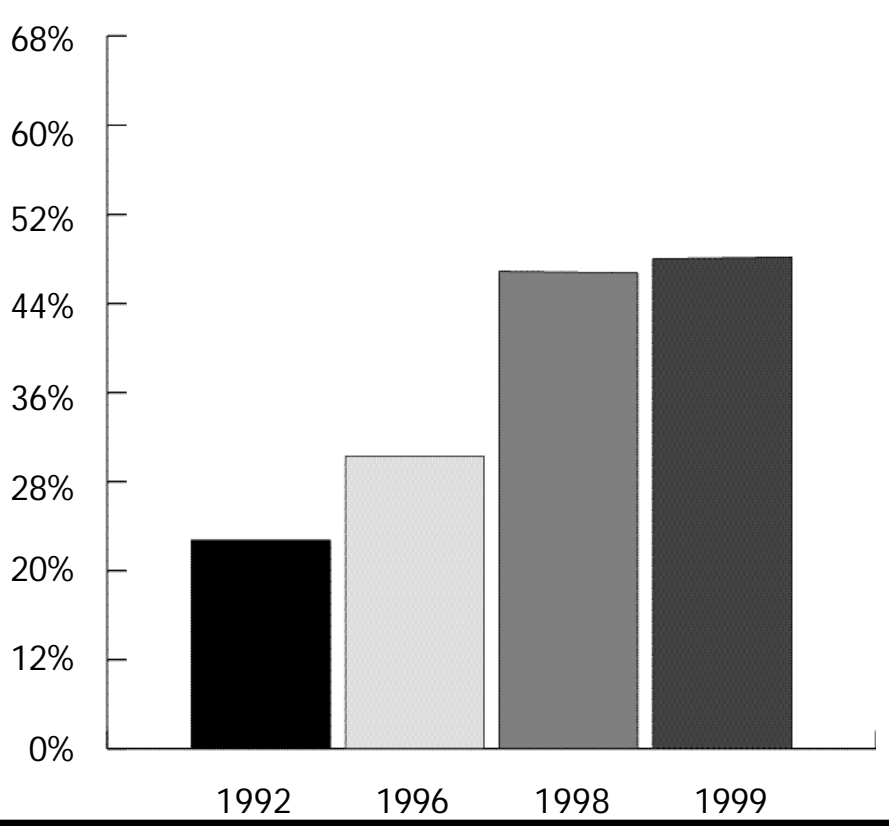

Source: Data from SM G Marketing Group, Inc., Chicago, IL, as published in M anaged Care Digest Series 2000. Parsippany, NJ: Aventis Pharmaceuticals, 2000.

HMO Non-Medicare Members

\begin{tabular}{l|c|c|c|c|c|c}
\hline & \multicolumn{2}{|c|}{$\begin{array}{c}\text { Prescriptions } \\
\text { per Member }\end{array}$} & $\begin{array}{c}\text { Average Ingredient } \\
\text { Cost }\end{array}$ & \multicolumn{2}{c|}{$\begin{array}{c}\text { Pharmacy Premium } \\
\text { PM PM }\end{array}$} & $\begin{array}{c}\text { Pharmacy as a } \\
\text { Percentage of Total } \\
\text { Operating Expenses }\end{array}$ \\
\hline & Non-Medicare & Medicare & & Individual & Family & \\
\hline 1988 & 4.9 & -- & $\$ 11.50$ & $\$ 7.40$ & $\$ 18.47$ & $10.0 \%$ \\
\hline 1990 & 5.7 & -- & $\$ 15.10$ & $\$ 9.40$ & $\$ 23.80$ & $9.0 \%$ \\
\hline 1994 & 6.0 & 16.5 & $\$ 21.54$ & $\$ 12.26$ & $\$ 31.28$ & $10.4 \%$ \\
\hline 1998 & 7.0 & 17.1 & $\$ 28.20$ & $\$ 16.98$ & $\$ 42.49$ & $13.6 \%$ \\
\hline 1999 & 7.1 & 18.1 & $\$ 29.35$ & $\$ 17.43$ & $\$ 43.32$ & $13.4 \%$ \\
\hline
\end{tabular}

Note: PMPM is per member per month.

Source: Data from SM G Marketing Group, Inc., Chicago, IL., as published in Managed Care Digest Series 2000. Parsippany, N J: Aventis Pharmaceuticals, 2000.

Medicare members correlates with decreased hospitalization and shorter length-of-stay; drug expenditures for Medicare patients increased only slightly during the same period when hospital admis- sions increased for this population.

Despite these opposite trends in the use of pharmaceuticals and acute care, pharmacies have come under intense scrutiny because their budgets have increased. In an effort to manage costs while striving for good care, pharmacies have used formularies, drug-utilization review (DUR), and pharmacy benefit managers. About $97 \%$ of HMO plans used formularies in 1999, with 
Trends in Managed Care Pharmacy: Preparing for the Future

\begin{tabular}{l|c|c|c|c|c|c|c|c}
\hline & \multicolumn{1}{|c|}{ HMO Utilization, 1998-1999 } & \multicolumn{3}{c}{$\begin{array}{c}\text { Average } \\
\text { Lospital Days per } \\
\mathbf{1 , 0 0 0} \text { Members }\end{array}$} & \multicolumn{2}{c|}{$\begin{array}{c}\text { Physician Encounters } \\
\text { per Member }\end{array}$} & \multicolumn{2}{c}{$\begin{array}{c}\text { Ambulatory Visits } \\
\text { per Member }\end{array}$} \\
\hline Payor Type & $\mathbf{1 9 9 8}$ & $\mathbf{1 9 9 9}$ & $\mathbf{1 9 9 8}$ & $\mathbf{1 9 9 9}$ & $\mathbf{1 9 9 8}$ & $\mathbf{1 9 9 9}$ & $\mathbf{1 9 9 8}$ & $\mathbf{1 9 9 9}$ \\
\hline Non-M edicare & 210.5 & 200.0 & 3.7 & 3.6 & 3.9 & 3.0 & 2.0 & 1.6 \\
\hline Medicare & $1,382.5$ & $1,328.8$ & 5.6 & 5.5 & 12.0 & 7.2 & 3.3 & 2.6 \\
\hline Medicaid & 402.0 & 394.8 & 3.5 & 3.5 & 4.3 & 3.4 & 1.8 & 1.6 \\
\hline Overall Average & $\mathbf{2 6 1 . 6}$ & $\mathbf{2 2 2 . 8}$ & $\mathbf{3 . 9}$ & $\mathbf{3 . 9}$ & $\mathbf{4 . 3}$ & $\mathbf{3 . 4}$ & $\mathbf{2 . 0}$ & $\mathbf{1 . 8}$ \\
\hline
\end{tabular}

Source: Data from SM G M arketing Group, Inc., Chicago, IL, as published in Managed Care Digest Series 2000. Parsippany, NJ: Aventis Pharmaceuticals, 2000.

\section{Integrated System Trends}

\section{Number of Systems by Delivery Components per System, 1995-1999}

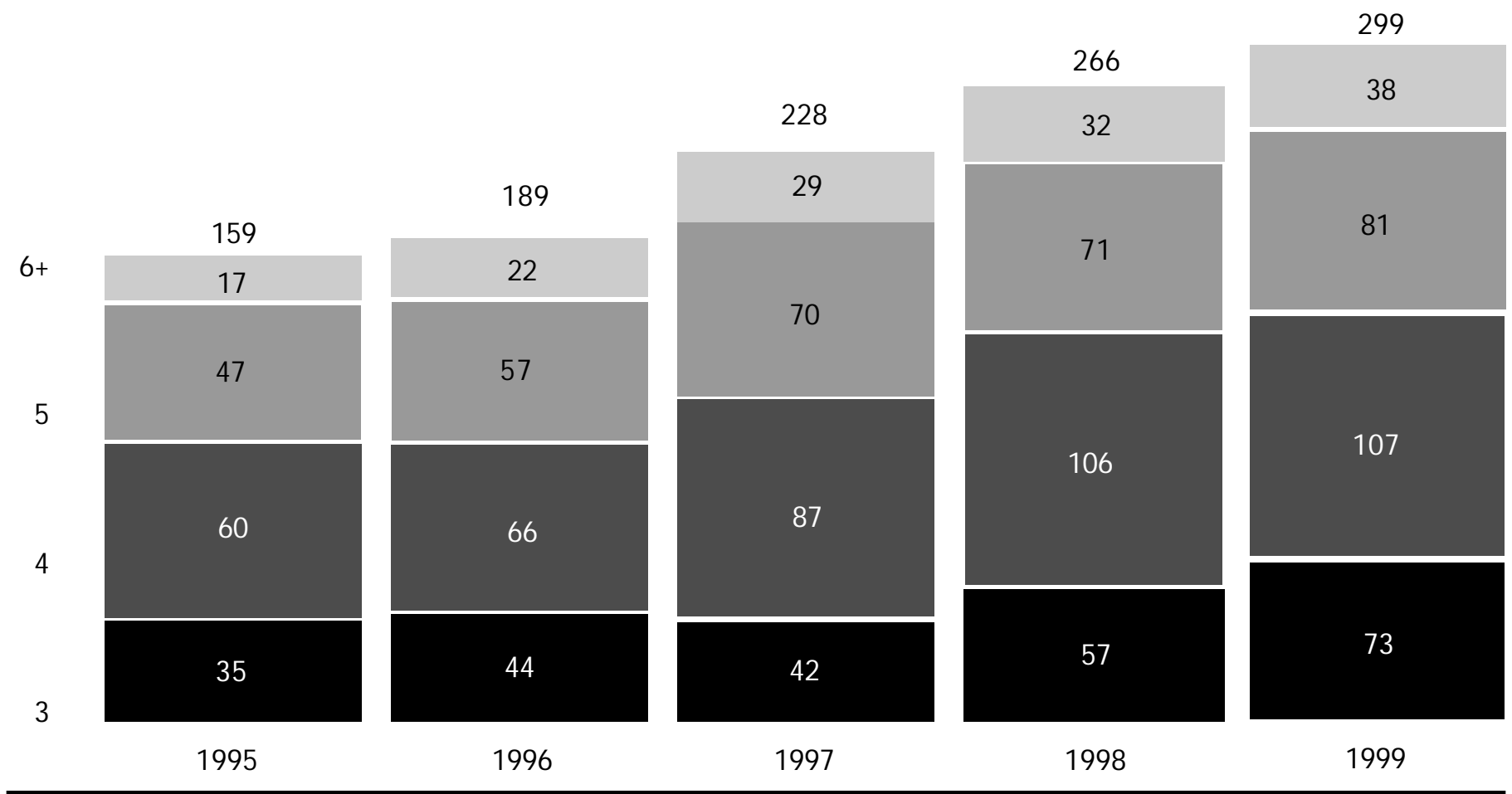

Source: Data from SM G Marketing Group, Inc., Chicago, IL, as published in Managed Care Digest Series 2000. Parsippany, N J: Aventis Pharmaceuticals, 2000.

nearly half being closed. Similarly, $94 \%$ used DUR. Many HMOs allow physicians to override formularies $(70.5 \%)$, but $81 \%$ of prescriptions were still filled within formularies in 1999.

\section{Integration of Managed Care}

Increased utilization and costs have also led to changes in the way MCOs have organized themselves. Significantly, there has been a steady increase in the degree of integration in health care systems over the past five years (see Figure 3, above).
Integrated systems are designed to improve the delivery of care by coordinating various health care components, such as outpatient and inpatient hospital services. Over the long term, they provide real opportunities for coordination of phar- 
macy services. Highly integrated systemsincluding three or more health care components-are also likely to assume more financial risk. Integrated systems have the potential to offer better data integration between health care sectors, although full potential of data integration to improvecare and reduce costs has not yet been realized.

\section{Changing Roles of Providers}

A shift in traditional roles for health care providers has also occurred in the past decade. The role of physician as primary decision maker has changed: Today physicians face more responsibility, more time pressure, and a more challenging economic environment. Patients are becoming more aggressive in their search for and use of information as they take on more responsibility for their own health care choices and outcomes. These factors have contributed to a fundamental change in the physician/patient relationship and the potential for tension among health plan, provider, and patient.

Pharmacists face similar changes in their roles. Prior to the advent of managed care, pharmacists functioned solely as heal th care providers, interacting directly with patients and physicians. The creation of managed care formularies and their implementation has added complexities to the pharmacist's role. Constant and ever-increasing scrutiny of pharmacy budgets has resulted in new administrative responsibilities and challenges for pharmacists, who must now increasingly focus on total pharmacy cost management as well as patient care. Pharmacists must also continually examine and confirm the value of pharmaceutical interventions in the broader continuum of care. Essentially, an entirely new discipline of pharmacy business management has resulted, and new skills are required, changing the nature of the pharmacist's job.

\section{Health Care Challenges}

The trends enumerated have resulted in important challenges that pharmacy professionals must address to ensure that managed care successfully meets patient needs while keeping costs manageable. The changing health care needs of an evolving member population demand new approaches to providing care. Pharmacy must be allowed to take a more direct and active role across the continuum of care. Changing technology and products require new criteria to determine appropriateness, not just efficacy and safety.

Cost concerns are also increasing. Specifically, increases in pharmacy expenditures have led to greater budget scrutiny and demands for proof of value. Although increased pharmacy expenditures for drug therapy have coincided with decreased hospital admissions and fewer days in the hospital, impact on total cost of care is not always clear, and may be impossible to measure.

Databases within each system component are often incompatible, making accurate analysis of total organizational costs extremely difficult. While some compatibility may exist between outpatient and inpa tient databases, pharmacy data is too often not integrated into the system. This information gap may represent the greatest single challenge facing $\mathrm{MCO}$ s because it affects their ability to provide the best possible care in a fiscally responsible manner. Poor data integration also affects an organization's ability to accurately assess systemwide costs of care and to find opportunities to realign resources or reduce costs. It may also put departments within the same health system in conflict by focusing on individual budgets rather than on organizational goals. More importantly, poor data integration means lost opportunities to improve patient care. Finally, it may put MCOs unnecessarily at financial odds with their providers or with their customers (employers) and members. On the other hand, a fully-integrateddata approach allows the entire system to analyze costs, risks, and rewards from an organizational viewpoint. Health plans must be able to compare effectiveness within their own organizations as well as compare themselves with other health plans.

\section{- Implications for Pharmacists}

The key to progress in clinical and cost outcomes is to use available data on member populations and their care conditions, services, and products to determine where these outcomes can be improved and costs can be managed more effectively. The first step in achieving this goal is to refocus efforts on patient needs, essentially promoting a "patient-centric" environment that supports improved physician/patient relationships. One way pharmacists can approach this is to identify particular member populations and specific information about pharmacotherapy as an early warning system regarding patients whose conditions can result in emergency room visits, hospital admissions, and high costs. Pharmacy personnel can intervene to ensure optimal care and prevent unnecessary services; they can learn whether various intervention strategies are working and should be deployed on a wider basis. Thus, pharmacists can play a pivotal role in helping MCOs support better clinical and cost outcomes by using their data to benchmark performance, even if an ideal, fully integrated data system is not in place.

Although pharmacy departments may be excluded from system-integration efforts, pharmacists can avoid the "silo effect" by taking the initiative to work with their organization's information systems department to obtain data. The lessons of system integration and structured change in managed care come together in a focused strategy directed at key problems. However, it is also vital to focus on interventions that show near-term cost benefits. Some long-term preventive initiatives may be better candidates for educational programs because of the asymptomatic nature of the condition and the fact that results might not be obvious for many years.

\section{Asthma}

Asthma is one of the most common chronic diseases, causing significant morbidity and accounting for total annual costs of up to $\$ 11.3$ billion in 1998. ${ }^{2}$ The increased incidence of asthma causes concern for all health systems. Between 
1980 and 1994, the prevalence of asthma per 1,000 persons increased $75 \%$; among children up to four years of age, asthma increased $160 \%$. $^{3}$

Standard treatment regimens recommend the use of inhaled corticosteroids as primary therapy in patients with moderate or severe chronic asthma and beta2agonists with or without corticosteroids for acute asthma. ${ }^{4}$ Regular adherence to an asthma treatment plan should reduce the need for acute care. Pharm-acists can determine whether chronic asthma is being treated adequately within a health system by requesting data for a subset of patients who have been diagnosed as having asthma and then cross-referencing it with patients who are prescribed crisis medications. Further analysis of specialist visits for allergy and asthma care, emergency room visits, and hospitalizations for this subset of asthma patients is likely to help the pharmacist identify patients who require more emergency care than would be necessary if a regular course of asthma drugs were used. Comparing the costs of care for patients who do not follow asthma treatment guidelines with those who do provides an opportunity for intervention and a likely cost reduction within a short period of time. Because pediatric patients are more likely than adult patients to require emergency asthma care, the model can even be used to identify more specific interventions based on plan demographic characteristics.

\section{Type-2 Diabetes}

As with asthma, the incidence of type-2 diabetes has continued to climb, especially among older persons and those who are obese. ${ }^{4}$ The increase in diabetes is of great concern to health plans because it is also linked to significant increases in morbidity from heart disease. Pharmacists can create a model to review appropriate intervention by analyzing the subset of diabetes patients using insulin. Inappropriate or unusual numbers of insulin refills might indicate poor disease management, for example. Further exam- ination of records for acute care, hospital admissions, and length-of-stay will help identify patients who are not adequately controlled on current insulin regimens or who exhibit signs of comorbid conditions suggesting a need for preventive care and stricter monitoring.

\section{Deep Venous Thrombosis}

Antithrombotic agents such as coumadin and low-molecular-weight heparin are generally used to treat acute deep venous thrombosis (DVT). Patients who are on antithrombotic therapy are already at high risk because of the thrombosis itself, and anticoagulants require intensive management to prevent complications. Pharmacists may wish to pay particular attention to patients on such medications. Again, identifying patients using anticoagulants and monitoring hospital admissions, length-of-stay, and emergency room treatment, as well as the duration of treatment, may provide insights into better intervention strate gies. It is also possible to determine whether aggressive management may obviate the need for other services, such as surgery. Comparing outcomes for inpatient versus outpatient care, medical costs versus surgical costs, and prevention costs versus treatment can help the pharmacist develop a detailed model illustrating the optimum use of antithrombotic therapy.

\section{Integrating Pharmacy Data}

Each of these examples requires the pharmacist to work directly with the information systems department to request specific data from a subset of managed care patients with the goals of improving patient health and reducing the costs of care. The pharmacist can take a leadership role in creating intervention models that demonstrate the value of the pharmacy component. Essentially, pharmacists are in the truly unique position of having data from all points along the continuum of care and therefore having an organizational perspective.

\section{- Conclusion}

Increased managed care utilization and expected increases in chronic diseases due to the aging population suggest a strong need for a new paradigm that encourages closer integration among all sectors in a health care system. Better data sharing and a focus on total costs rather than specific department budgets may expose opportunities for cost savings. More important, using systemwide data can contribute to better patient care, but not at the expense of careful fiscal management.

Pharmacy departments are in a key position to effect change within managed care systems. Pharmacists should use the data at their disposal as well as external sources of information that identify trends in managed care to develop a targeted approach to improving patient care and reducing costs. Data-based efforts to identify target populations, target conditions, and key predictors of cost and inappropriate utilization represent major opportunities for health plans. The pharmacy department, using pharmacy data, should be the team leader in this effort.

\section{REFERENCES}

1. Managed care digest series 2000. Parsippany, NJ: Aventis Pharmaceuticals, 2000.

2. National Heart, Lung, and Blood Institute, N ational Institutes of Health. Data fact sheet: asthma statistics. Available at www.nhlbi.nih.gov/ health/prof/lung/asthma/am_fa99/asthfcts.htm.

3. Centers for Disease Control and Prevention. CDC surveillance summaries. MMWR 1998; 47 (SS-1).

4. Joint Task Force on Practice Parameters in Allergy, Asthma and Immunology. Practice parameters for the diagnosis and treatment of asthma. Allergy Clin Immunol 1995; 96 (5 Pt 2): S707-870. 